IMECE2005-80227

\title{
USING PHASED ARRAY TECHNOLOGY AND EMBEDDED ULTRASONIC STRUCTURAL RADAR FOR ACTIVE STRUCTURAL HEALTH MONITORING AND NONDESTRUCTIVE EVALUATION
}

\author{
Lingyu $\mathrm{Yu}, \mathrm{PhD}$ candidate \\ Mechanical Engineering Department, University of \\ South Carolina \\ Columbia, SC 29208, yu3@engr.sc.edu
}

\author{
Victor Giurgiutiu, Professor \\ Mechanical Engineering Department, University of \\ South Carolina \\ Columbia, SC 29208, giurgiut@engr.sc.edu
}

\begin{abstract}
The embedded ultrasonic structural radar (EUSR) was developed based on phased array technology. It can interrogate large structural areas from a single location using ultrasonic guided Lamb and Rayleigh waves generated by tuned piezoelectric wafer active sensors (PWAS) that are permanently attached to the structure. This paper brings together several aspects of the implementation and application of EUSR to structural damage detection: (a) improving the near field damage detection; (b) designing optimized phased-array patterns; (c) designing a mini phased array for compact structures with complicated geometries and multiple boundaries.

Firstly, we deduced a generic formulation for phased array directional beamforming using the exact traveling waves formulation without the limiting parallel-rays assumption used by other investigators. This algorithm has been implemented in the EUSR LabVIEW program and its performance has been verified through simulation and experimental tests. Secondly, we studied the beamforming and lobe steering characteristics of a 1-D linear array design. The influence of several geometry parameters was discussed in order to achieve the optimal directionality, including the number of sensors in the phased array, the spacing between adjacent sensors, and the steering direction angles. Extensive simulation studies have shown that the well-behaved directional beamforming can be achieved with judicious array design. Proof-of-concept experiments for testing these results have also been set up and the preliminary results are confirming the effectiveness of our approach. Thirdly, we investigated the possibility of applying the EUSR phased array method to compact specimens and proposed the design of a mini phased array. Laboratory experiments have been carried out to prove the successful implementation of this concept. Finally, the paper ends up with discussions and conclusions regarding the beamforming, optimization and implementation of the PWAS phased arrays, as well as suggestions for further work.
\end{abstract}

\section{INTRODUCTION}

Phased arrays are made of multiple piezoelectric elements excited by predetermined time-delayed signals to generate structural interference patterns. By properly adjusting the time delays, phased array can phase steer and focus the ultrasonic beams at certain direction. Some of the advantages of phased arrays over conventional ultrasonic transducers include high inspection speed, flexible data processing capability, improved resolution, and the capability of scanning without requiring mechanical movement, i.e., dynamic beam steering and focusing [1]. The backscattered ultrasonic signals can be analyzed and then mapped into an image.

Current ultrasonic inspection of thin wall structures (e.g., aircraft shells, storage tanks, large pipes, etc.) is a time consuming operation that requires meticulous through-thethickness C-scans over large areas. One method to increase the efficiency of thin-wall structures inspection is to utilize guided waves (e.g., Lamb waves) instead of the conventional pressure waves $[2,3]$. Guided waves propagate along the mid-surface of thin-wall plates and shallow shells $[4,5]$. They can travel at relatively large distances with very little amplitude loss and offer the advantage of large-area coverage with a minimum of installed sensors. Guided Lamb waves have opened new opportunities for cost-effective detection of damage in aircraft structures, and a large number of papers have recently been published on this subject. The use of guided waves in conjunction with phase-array principles has proliferated widely in recent years due to its obvious benefits. However, an important roadblock on the way towards the utilization of these techniques in aircraft structural health monitoring is that the conventional ultrasonic transducers used for guided wave and phased-array applications are bulky and expensive, thus making their use in wide-area structural health monitoring expensive and problematic. Hence, a different type of sensors than the conventional ultrasonic transducers is required for the SHM systems. Piezoelectric wafer active sensors (PWAS) are small, non-intrusive, inexpensive piezoelectric wafers that are 
permanently affixed to the structure and can actively interrogate the structure. PWAS are non-resonant devices with wide band capabilities. They can be wired into sensor arrays and connected to data concentrators or wireless communicators [6]. As active sensors, PWAS can be used as both transmitters and receptors of elastic waves. An important characteristic of PWAS is their omnidirectionality. This makes PWAS better suited for large area inspection using phased array electronic automated scanning (Conventional ultrasonic transducers for guided-wave generation are unidirectional, and hence can only scan by mechanically rotating the transducer.).

\section{GENERIC FORMULATION FOR BEAMFORMING}

Phased array is a group of sensors located at distinct spatial locations in which the relative phases of the respective signals feeding the sensors are varied in such a way that the effective propagation pattern of the array is reinforced in a desired direction and suppressed in undesired directions [7]. That's to say, the phased array acts like a spatial filter attenuating all signals and saving those propagating from certain directions. Beamforming is the array signal processing algorithms that focus the array's signal capturing ability in a particular direction. Piezoelectric-wafer active sensors (PWAS) are small, non-intrusive, inexpensive piezoelectric wafers that are permanently affixed to the structure and can actively interrogate the structure [6]. They can be wired into sensor arrays and serve as active sensor for both generation and reception of elastic waves. We will deduce the general formula of PWAS phased array based on the delay-and-sum beamforming algorithm without the limiting parallel-rays assumption.

\subsection{Delay-and-sum Beamforming}

The idea of delay-and-sum beamforming is: if a propagating signal is present in an array's aperture, the sensor outputs, delayed by appropriate amounts and added together, reinforce the signal with respect to noise or waves propagating in different directions. The delays that reinforce the signal are directly related to the length of time it takes for the signal to propagate between sensors. Suppose there is a reflector at the point $P(\vec{r}, \varphi)$. We will consider the wavefield generated at $P(\vec{r}, \varphi)$ by an array of $M$ sensors, located at $\left\{\vec{s}_{m}\right\}, m=0, \ldots, M-$ 1. The origin of coordinate system coincides with the sensors phase center which is defined as the vector quantity $\frac{1}{M} \sum \vec{s}_{m}$. Hence, the origin is the point where

$$
\frac{1}{M} \sum \vec{s}_{m}=0
$$

The wave emitting from the $m^{\text {th }}$ sensor is $y_{m}(t)=f\left(\vec{r}_{m}, t\right)$ where $\vec{r}_{m}$ is the vector from the $m^{\text {th }}$ sensor to the reflector (Figure 1).

$\vec{\xi}$ is the unit vector that points from the origin to the target points $P(\vec{r}, \varphi)$ and $\vec{\xi}_{m}$ is the unit vector that points from the $m^{\text {th }}$ sensor to the target points $P(\vec{r}, \varphi)$.

The delay-and-sum beamforming consists of two steps: (1) applying a delay $\Delta_{m}$ and a weighting factor $w_{m}$ to the output of $m^{\text {th }}$ sensor; (2) summing up the output signals of $M$ sensors. This processing is defined as:

$$
z(t)=\sum_{m=0}^{M-1} w_{m} y_{m}\left(t-\Delta_{m}\right)
$$

$w_{m}$ is the weighting factor, designing for enhancing the beams' shape and reducing sidelobe levels. The delay $\left\{\Delta_{m}\right\}$ are adjusted to focus the array's beam on particular propagating direction $\vec{\xi}$. Importantly, the beamforming algorithm varies according to whether the reflector is located near to or far from the array.

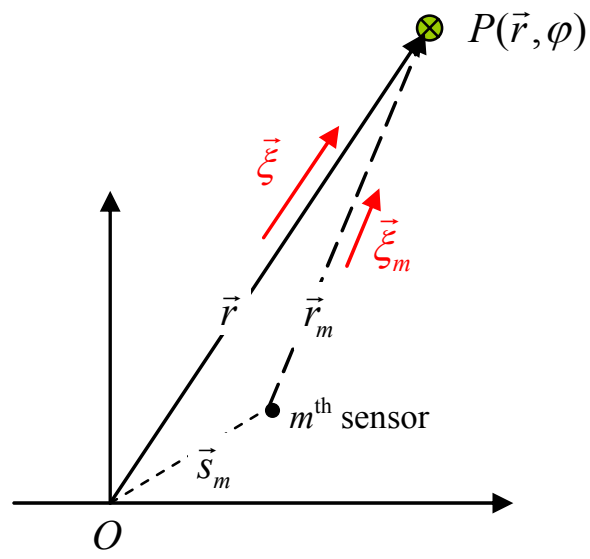

Figure 1: the geometry schematics of the $m^{\text {th }}$ sensor and the reflector at $P(\vec{r}, \varphi)$

If the reflector is close to the array, i.e., within near field (Figure 2a), the propagating wavefront is curved (circular wavefront) with respect to the array and the wave propagating directions are dependent on the location of each sensor. When the reflector is far away from the array, i.e., in the far field to the array (Figure $2 \mathrm{~b}$ ), the propagation directions of individual waves approximate parallel to each other, that's to say, $\vec{\xi}_{m} \approx \vec{\xi}$, and the propagating field within the array consists of plane waves. (a)

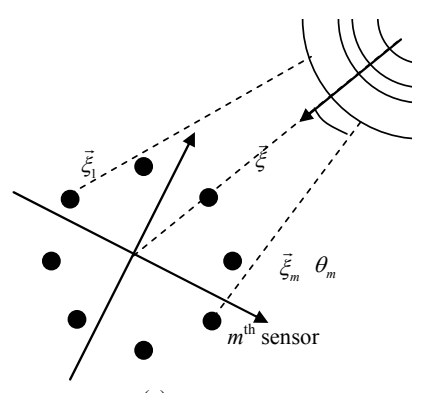

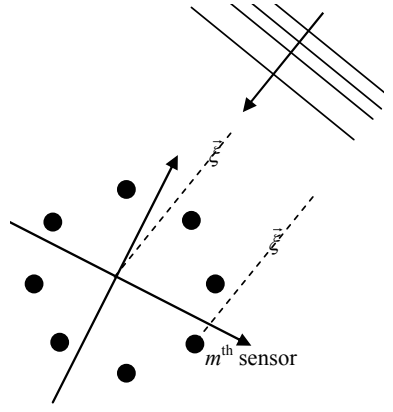

(b)
Figure 2: beamforming in near- and far- field to the array: (a) near field; (b) far field [7]

Consider a particular direction of wave propagation $\vec{\xi}$ pointing to the point $P(\vec{r}, \varphi)$ from the origin (phase center) as shown in Figure 1. For far-field situation, it will also be the propagating directions for all the plane waves, regardless of the location of the sensors. But for near-field situation, the propagation directions vary from sensor to sensor (Figure 2a). We need to 
define direction vectors for each senor, say, $\vec{\xi}_{m}$ for $m^{\text {th }}$ sensor. As indicated in Figure 1, we have:

$$
\begin{gathered}
\vec{\xi}=\frac{\vec{r}}{|\vec{r}|} \\
\vec{\xi}_{m}=\frac{\vec{r}_{m}}{\left|\vec{r}_{m}\right|}, \vec{r}_{m}=\vec{r}-\vec{s}_{m}
\end{gathered}
$$

$\vec{s}_{m}$ is the location of the $m^{\text {th }}$ sensor.

\subsection{Beamforming for plane waves}

Assume a far-field source emitting a plane wave $s(t)$ propagating in the direction $\vec{\xi}$ to the array. The wavefront arriving at the array is:

$$
f(\vec{r}, t)=s(t-\vec{\alpha} \cdot \vec{r})
$$

where $\vec{\alpha}=\vec{\xi} / c$ and $c$ is the wave propagation speed. The $m^{\text {th }}$ sensor spatially sampled in this wave field yields:

$$
y_{m}(t)=s\left(t-\vec{\alpha}_{m} \cdot \vec{s}_{m}\right)
$$

with $\vec{\alpha}_{m}=\vec{\xi}_{m} / c$. By applying both a weighting factor and a time delay to it, we have:

$$
z_{m}(t)=w_{m} \cdot s\left(t-\vec{\alpha}_{m} \cdot \vec{s}_{m}-\Delta_{m}\right)
$$

And then sum up the M sensors:

$$
z(t)=\sum_{m=0}^{M-1} z_{m}(t)=\sum_{m=0}^{M-1} w_{m} s\left(t-\vec{\alpha}_{m} \cdot \vec{s}_{m}-\Delta_{m}\right)
$$

From equation (8) we see, if we make:

$$
\Delta_{m}=-\vec{\alpha}_{m} \cdot \vec{s}_{m}
$$

we will have:

$$
z(t)=s(t) \cdot \sum_{m=0}^{M-1} w_{m}
$$

Hence, the beamforming signal is a constant times enhancement of the original signal. By applying proper time delay (using formula in equation (9)), the array's beamforming can be steered to a certain direction $\vec{\xi}$, i.e.:

$$
z(\vec{r}, t)=\sum_{m=0}^{M-1} w_{m} f\left(\vec{r}_{m}, t-\Delta_{m}\right)
$$

\subsection{PWAS phased array beamforming formulation}

Generally, the wavefront at a point $\vec{x}$ away the PWAS source is:

$$
f(\vec{x}, t)=\frac{A}{\sqrt{|\vec{x}|}} e^{i(\omega t-\vec{k} \cdot \vec{x})}
$$

Supposing we have an array of $M$ PWAS installed at $\left\{\vec{s}_{m}\right\}$ sending out signals in the direction of $\left\{\vec{\xi}_{m}\right\}$ to $P(\vec{r}, \varphi)$ respectively, using equation (11) the synthetic wavefront $z(\vec{r}, t)$ at the point $P(\vec{r}, \varphi)$ with time delay and weighting is:

$$
z(\vec{r}, t)=\sum_{m=0}^{M-1} w_{m} f\left(\vec{r}_{m}, t-\Delta_{m}\right)
$$

The origin is where $\sum \vec{s}_{m}=0$. Other variables are defined as:

$$
\left\{\begin{array}{l}
r=|\vec{r}| \\
\vec{\xi}=\frac{\vec{r}}{|\vec{r}|}=\frac{\vec{r}}{r}
\end{array}\right.
$$

and

$$
\left\{\begin{array}{l}
r_{m}=\left|\vec{r}_{m}\right|=\left|\vec{r}-\vec{s}_{m}\right| \\
\vec{\xi}_{m}=\frac{\vec{r}_{m}}{\left|\vec{r}_{m}\right|}=\frac{\vec{r}_{m}}{r_{m}}=\frac{\vec{r}_{m}}{\left|\vec{r}-\vec{s}_{m}\right|}
\end{array}\right.
$$

The wavenumber of the wave propagating in the direction of $\vec{\xi}_{m}$ is defined as:

$$
\vec{k}_{m}=\vec{\xi}_{m} \cdot \frac{\omega}{c}
$$

where $\omega$ is the wave's temporal frequency. Using the notations defined in equation (13), (14) and (15), the wavefront from the $m^{\text {th }}$ sensor arriving at the point $P(\vec{r}, t)$ can be written as:

$$
f\left(\vec{r}_{m}, t\right)=\frac{A}{\sqrt{r_{m}}} e^{i\left(\omega t-\vec{k}_{m} \cdot \vec{r}_{m}\right)}
$$

\subsubsection{Far-field beamforming using the parallel ray} approximation

As discussed in 1.1, if a reflector is far away from the array, the propagation directions of individual waves can be considered being parallel to each other, i.e.,. $\vec{\xi}_{m} \approx \vec{\xi}$. Hence,

$$
\vec{k}_{m} \approx \vec{\xi} \cdot \frac{\omega}{c}=\vec{k}
$$

and

$$
\sqrt{r_{m}} \approx \sqrt{r}
$$

Equation (16) becomes:

$$
\begin{aligned}
f\left(\vec{r}_{m}, t\right) & \approx \frac{A}{\sqrt{r}} e^{\left\{i \omega\left(t-\frac{\vec{\xi} \cdot\left(\vec{r}-\vec{s}_{m}\right)}{c}\right)\right\}} \\
& =\frac{A}{\sqrt{r}} e^{\left\{i \omega\left(t-\frac{r}{c}+\frac{\vec{\xi} \cdot \vec{s}_{m}}{c}\right)\right\}} \\
& =\frac{A}{\sqrt{r}} e^{i \omega\left(t-\frac{r}{c}\right)} \cdot e^{\left\{i \frac{\omega}{c} \vec{\xi} \cdot \vec{s}_{m}\right\}} \\
f\left(\vec{r}_{m}, t\right) & =f\left(\vec{r}, t-\frac{r}{c}\right) \cdot e^{\left\{i \frac{\omega}{c} \vec{\xi} \cdot \vec{s}_{m}\right\}}
\end{aligned}
$$

In equation (19) the wavefront $f\left(\vec{r}_{m}, t\right)$ is only dependent on the exponential part. The synthetic waves of $M$ sensors with delaying and weighting therefore is:

$$
z(\vec{r}, t)=\sum_{m=0}^{M-1} w_{m} f\left(\vec{r}, t-\frac{r}{c}+\frac{\vec{\xi} \cdot \vec{s}_{m}}{c}+\Delta_{m}\right)
$$

If we adjust the time delay $\Delta_{m}$ in such a way that

$$
\Delta_{m}=-\frac{\vec{\xi} \cdot \vec{s}_{m}}{c}
$$


$z(\vec{r}, t)$ becomes:

$$
z(\vec{r}, t)=f\left(\vec{r}, t-\frac{r}{c}\right) \cdot \sum_{m=0}^{M-1} w_{m}
$$

When $w_{m}=1$, the synthetic signal is $M$ times reinforced $f(\vec{r}, t)$.

\subsubsection{Near-field beamforming}

Within the field near to the array, the parallel ray approximation is not valid. The exact traveling waves are used for the beamforming formulation.

$$
\begin{gathered}
f\left(\vec{r}_{m}, t\right)=\frac{A_{m}}{\sqrt{r_{m}}} e^{i \omega\left(t-\frac{\vec{\xi}_{m} \cdot \vec{r}_{m}}{c}\right)} \\
f\left(\vec{r}_{m}, t\right)=f\left(\vec{r}, t-\frac{r}{c}\right) \frac{1}{\sqrt{r_{m} / r}} e^{i \omega\left(\frac{r-r_{m}}{c}\right)}
\end{gathered}
$$

The synthetic signal $z(\vec{r}, t)$ is:

$$
z(\vec{r}, t)=\sum_{m=0}^{M-1} \frac{w_{m}}{\sqrt{r_{m} / r}} f\left(t-\frac{r}{c}+\frac{r-r_{m}}{c}+\Delta_{m}\right)
$$

If we have

$$
\begin{aligned}
\Delta_{m} & =-\frac{r-r_{m}}{c} \\
w_{m} & =\sqrt{r_{m} / r}
\end{aligned}
$$

equation (25) becomes:

$$
z(\vec{r}, t)=M f\left(\vec{r}, t-\frac{r}{c}\right)
$$

We see, the wavefront at the point $P(\vec{r}, \varphi)$ is still $M$ times reinforcing of the sending out signal.

\section{PHASED ARRAY IMPLEMENTATION AND OPTIMIZATION, THE EMBEDDED ULTRASONIC STRUCTURAL RADAR USING LAMB WAVES}

Embedded Ultrasonics Structural Radar (EUSR) is a new concept and methodology for in-situ nondestructive evaluation (NDE) of thin-wall structures. EUSR utilizes (a) a PWAS array embedded onto the structure; and (b) electronic modules for signal transmission/reception, processing, and interpretation. The principle of operation of the EUSR is derived from two general principles: (1) The principle of guided Lamb wave generation with piezoelectric wafer active sensors (PWAS); (2) The principles of conventional phased-array radar. It is assumed that each active sensor in the array acts as an omnidirectional transmitter/receiver.

\subsection{1-D linear PWAS phased array simulation}

The EUSR methodology was developed as an extension of some previous work on the Lamb wave propagation NDE, based on the study of the Lamb wave characteristic [6]. Each element in the PWAS array plays the role of both transmitter and receiver. The responses of the structure to all the excitation signals are collected. Adopted from the beamforming process currently used in phased-array radar applications, the EUSR algorithm applies an appropriate delay to each signal in the data set to make them all focus on a certain direction. When this direction changes within $0^{\circ}$ to $180^{\circ}$ range, a virtual scanning beam will form and a large area of the structure can be interrogated. Figure 3 is the schematics of a 1-D linear array consisting of $M$ PWAS, equally separated by $d$. The span of the array is $D$ with $D \approx(M-1) d$. The coordinate origin is defined to be the phased center according to equation (1). The array is distributed along the $x$-axis and centered about $y$-axis. The location of $m^{\text {th }}$ vector is:

$$
\vec{s}_{m}=\left(\left(m-\frac{M-1}{2}\right) d, 0\right)
$$

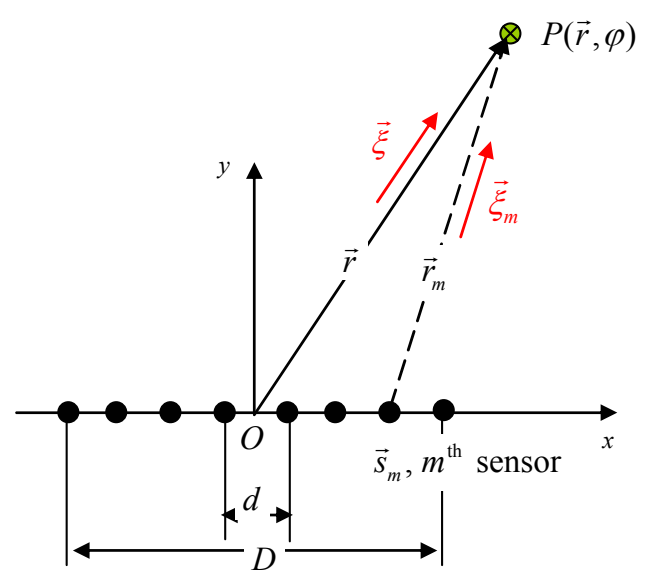

Figure 3: the schematics of an $M$-element 1-D linear PWAS phased array. The coordinate origin is located at the phased center, i.e., the center of the array.

According to the Lamb wave field formula shown as equation (12), the synthetic wavefront at the point $P(\vec{r}, \varphi)$ is:

$$
D S\left(\theta, \frac{d}{r}, \frac{d}{\lambda}, M\right)=\frac{1}{M} \cdot \sum_{m=0}^{M-1} \frac{\exp \left\{j 2 \pi \frac{d}{\lambda} / \frac{d}{r}\left(1-r_{m}\right)\right\}}{\sqrt{r_{m}}}
$$

where

$$
r_{m}=\left|\vec{r}-\vec{s}_{m}\right|
$$

From equation (30) we see, the beamforming $D S$ at a single angle $\theta$ is controlled by the ratio of $d / r$, the ratio of $d / \lambda$, and the number of sensors in the array.

After the direct beamforming, we then apply the time delay and weighting to each component in order to steer the beam to form at certain direction $(\theta)$. The delay-and-sum beamforming is then:

$$
D S\left(\theta, \theta_{0}, \frac{d}{r}, \frac{d}{\lambda}, M\right)=\frac{1}{M} \sum_{m=0}^{M-1} \frac{\exp \left\{j 2 \pi \frac{d}{\lambda} / \frac{d}{r}\left(1-r_{m}-\delta_{m}\left(\theta_{0}\right)\right)\right\}}{\sqrt{r_{m}}}
$$

with

$$
\delta_{m}\left(\theta_{0}\right)=1-r_{m}\left(\theta_{0}\right)
$$


The simulation results of original beamforming (without delay and weighting) and the directional beamforming at $\theta_{0}=45^{\circ}$ is shown in Figure 4, with $\mathrm{M}=8, d / \lambda=0.5$, and $d / r=1 /(M-1)^{*} 10$. The solid beam at $90^{\circ}$ position is the original beamforming and the dash beam at $45^{\circ}$ position is the delay and weighted beamforming.

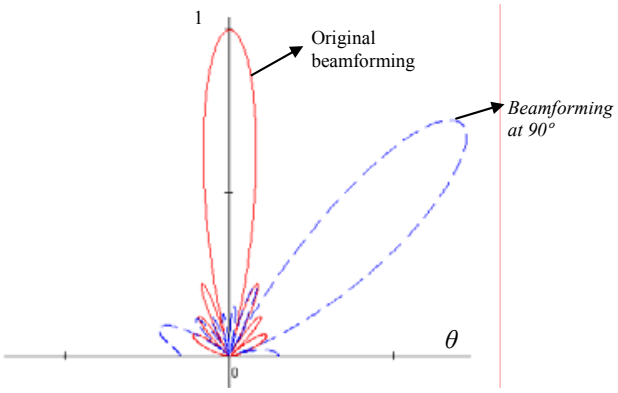

Figure 4: the original beamforming and directional beamforming at $45^{\circ}$ of an 8 PWAS linear phased array with $d / \lambda=0.5, d / r=1 /(M-1) \cdot 10$.

\subsection{Optimization of the PWAS array}

We have found out through the equation (32) that the directional beamforming at any angle $\theta_{0}$ depends on several parameters: (1) the spacing between adjacent sensors $d$; (2) the number of sensors $M$; (3) steering angle $\theta_{0}$. Among these, the effect of spacing $d$ is represented by the two ratios $d / \lambda$ and $d / r$. Our previous work on the Lamb wave propagation NDE [6] has found out that at the ratio $a / \lambda=0.5$ where $a$ is the width of the PWAS, PWAS will generate optimal excitation. Considering the point-wise assumption in the phased array application and the relation $d=a+\Delta \approx a$ ( $\Delta$ is the gap between sensors), this relation is represented as $a / \lambda=0.5$ in our discussion. This ratio still follows the spatial sampling theorem that $d$ should be smaller or equal to the half of wavelength ${ }^{2} . \lambda$ is the wavelength of the excitation signal (equal to $c / f$ ) and changeable by frequency tuning.

\subsubsection{Ratio of $d / r$}

The ratio of $d / r$ is related to the definition whether a point is located in the far or near field to the phased array. The far field can be considered as the area which distance to the phase center is much larger than the size of the array, i.e., the span $D$ of the array. Conventionally, the area can be treated as a far field if $D / r>5$. The near field can be anywhere below this ratio with the lower limit, "very close to the array" field is defined as $D / r$ $=1$ considering the existence of sidelobes. For our point-wise source, there is $D \approx(M-1) d$. Hence the ratio of $D / r$ can be transformed to $d / r$ using:

$$
d / r=D /(M-1) \cdot r
$$

Figure 5 shows the beamforming simulation of an 8-PWAS array at very close $(d / r=1 /(M-1) \cdot 1)$ field, near field $(d / r=$ $1 /(M-1) \cdot 2)$, and far field $(d / r=1 /(M-1) \cdot 5)$ to the array. From the simulation result we see, the directionality is best in the far field with small sidelobes. When getting closer to the array, the sidelobes increase in numbers and amplitudes. At the very close area to the area (where $D / r=1$ ), a large amount of energy leaks

\footnotetext{
${ }^{2}$ In order to avoid spatial aliasing, the ratio of $d / \lambda$ should be smaller of
} equal to 0.5 [7]. to the sidelobes from the mainlobe which results in the obvious decreasing of the mainlobe and increasing of sidelobes amplitude.

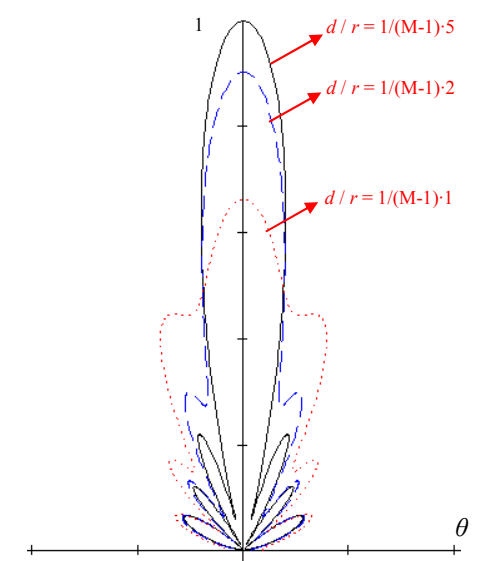

Figure 5: the beamforming of an 8 PWAS linear phased array (without delay) at different $d / r$ ratio with $d / \lambda=0.5$. The solid line is the beamforming for far field $(d / r=1 /(M-1) \cdot 5)$; dash line is for near field $(d / r=1 /(M-1) \cdot 2)$ and dot line for very near field $(d / r=1 /(M-1) \cdot 1)$ to the array.

\subsubsection{Number of sensors, $M$}

In order to achieve better directionality, finer mainlobe and smaller sidelobes are desired. Figure 6 shows the comparison results of the beamforming using 8 and 16 PWAS respectively with $d / r=1 /(M-1) \cdot 5$ (far field). It shows that with 16 sensors, we have a finer directional lobe and smaller sidelobes. More sensors will bring better directionality and fewer disturbances from the sidelobes.

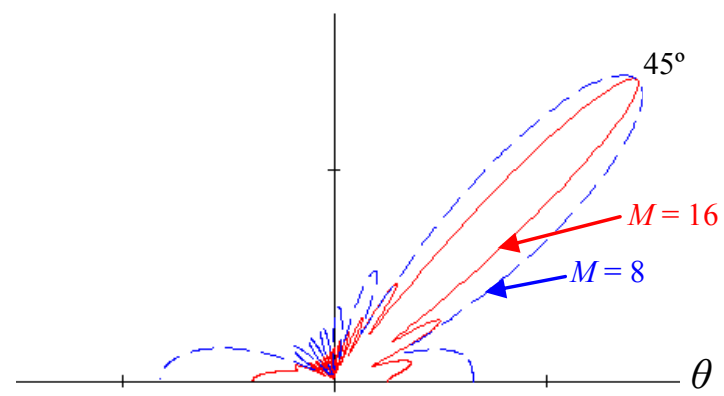

Figure 6: the beamforming of 8 PWAS array and 16 PWAS array at $45^{\circ}$, respectively with $d / r=1 /(M-1) \cdot 5$

\subsubsection{Steering angle $\theta_{0}$}

Steering angle $\theta_{0}$ is another factor that affects the beamforming. The beamforming at $0^{\circ}, 30^{\circ}, 60^{\circ}, 90^{\circ}, 120^{\circ}, 150^{\circ}$ using 8 PWAS is shown in Figure 7. Firstly, the beamforming are symmetric about $90^{\circ}$. Secondly, within $0^{\circ} \sim 90^{\circ}$ range, the beamforming loses its directionality at small angles such as $0^{\circ}$ and $30^{\circ}$ in the plot. However from $0^{\circ}$ to $30^{\circ}$, the directionality starts getting better. Lastly, when $\theta_{0}$ increases, the directionality is improved with sidelobes being suppressed (comparing $30^{\circ}$ with $60^{\circ}$ and $\left.90^{\circ}\right)$. 
Compared to the beamforming of 8 PWAS, the beamforming of 16 PWAS (Figure 8) shows better directionality not only at larger angles such as $60^{\circ}$ and $90^{\circ}$ (thinner directional mainlobes at corresponding $\theta_{0}$ ) but also at the lower angle $30^{\circ}$. At $30^{\circ}$, the beamforming of 16 PWAS array still keeps the directionality.

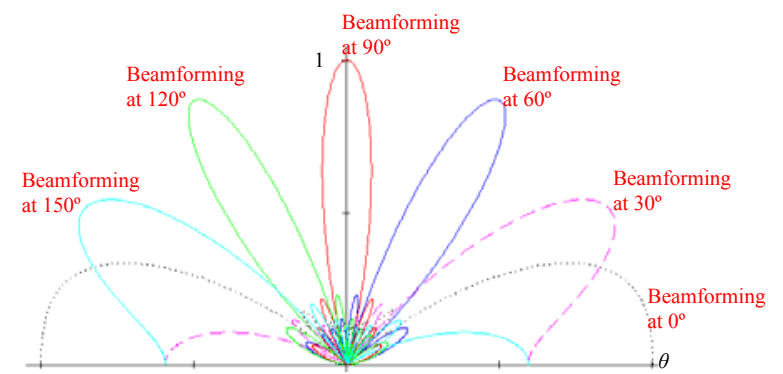

Figure 7: the beamforming of 8 PWAS array at $0^{\circ}, 30^{\circ}, 60^{\circ}, 90^{\circ}, 120^{\circ}$, $150^{\circ}$ with $d / r=1 /(M-1) \cdot 5$.

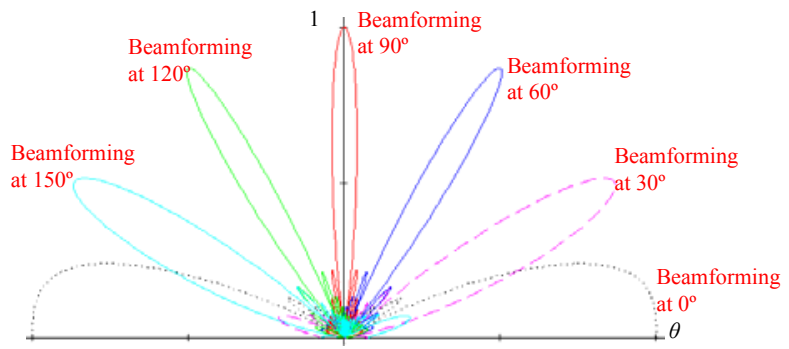

Figure 8: the beamforming of 16 PWAS array at $0^{\circ}, 30^{\circ}, 60^{\circ}, 90^{\circ}, 120^{\circ}$, $150^{\circ}$ with $d / r=1 /(M-1) \cdot 5$

The beamforming of 8 PWAS array with $d / r=1 /(M-1) \cdot 1$ at different angles are shown in Figure 9. Again, we see the beamforming gets better at larger $\theta_{0}$. However, the results in Figure 10 shows, at certain $\theta_{0}$, the ratio of $d / r$ will not affect the mainlobe, i.e., the directionality. Yet, the sidelobes strength is affected. It decreases with smaller $d / r$. But the difference between near field $(d / r=1 /(M-1) \cdot 2)$ and far field $(d / r=1 /(M$ 1) 5 or $d / r=1 /(M-1) \cdot 10)$ are almost negligible.

In summary, a well-behaved directional beamforming can be achieved with judicious array design according to our simulation test. In order to have good directionality at larger angular range, a large amount of sensors and/or smaller $d / r$ ratio are desired. However, we cannot increase the number of sensors indefinitely due to the wiring concerns.

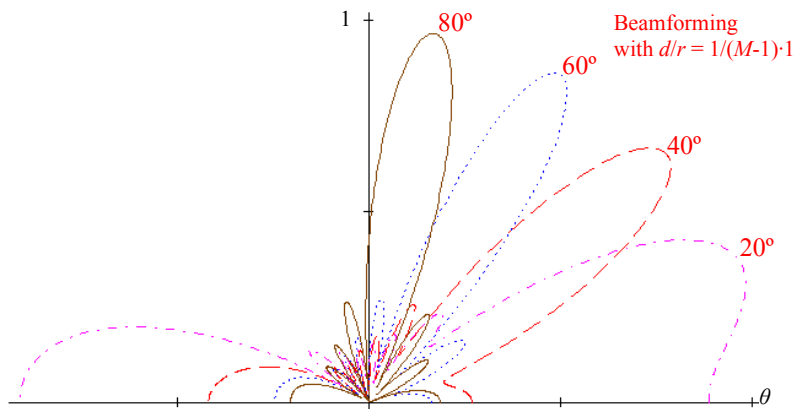

Figure 9: the beamforming of 8 PWAS array at $20^{\circ}, 40^{\circ}, 60^{\circ}, 80^{\circ}$ with $d / r=1 /(M-1) \cdot 1$

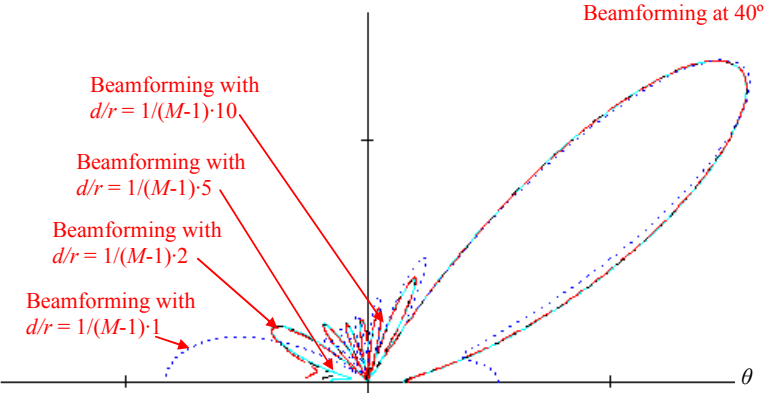

Figure 10: the beamforming of 8 PWAS array at $20^{\circ}$ with $d / r=1 /(M$ $1) \cdot 1,1 /(M-1) \cdot 2,1 /(M-1) \cdot 5$, and $1 /(M-1) \cdot 10$.

\subsection{Software implementation: the Embedded Ultrasonic Structural Radar (EUSR) software}

Pulse-echo method is used in the embedded ultrasonic structural radar system. A target is assumed at $P\left(r, \theta_{0}\right)$. The transmitting-receiving beamforming is sweeping the range in increasing angles $\theta$ and receiving an echo when $\theta=\theta_{0}$. The echo will be received on all sensors and applied with certain time delay for synchronization. Lamb waves can exist in a number of dispersive modes. However, through smoothed tone-burst excitation and frequency tuning, it is possible to confine the excitation to a particular Lamb wave mode, of carrier frequency $f_{c}$, wave speed $c$, and wave length $\lambda=c / f_{c}$. Hence, the smoothed tone-burst signal generated by one PWAS is of the form:

$$
s_{T}(t)=s_{0}(t) \cos 2 \pi f_{c} t
$$

where $s_{0}(t)$ is a short-duration smoothing window that is applied to the carrier signal of frequency $f_{c}$ between 0 and $t_{p}$ (Figure 11).

The principle of delay-and-sum beamforming is then applied to the PWAS-generated guided waves, assuming a uniform linear array of $M$ PWAS spacing at $d$, with each PWAS acting as a pointwise omni-directional transmitter and receiver. The total signal received at point $P\left(r, \theta_{0}\right)$ will be the amplified signal presented in equation (28). That's to say, there is an $M$ times increase in the signal strength with respect to a simple sensor. The receiving signal beamforming can be generated by treating the point $P$ as an omni-diectional souce at $\left(r, \theta_{0}\right)$ and the delay will be applied the the signals received at the $m^{\text {th }}$ sensor in the array. The synchronized receiving signal at all sensors then are achieved:

$$
s_{R}(t)=\frac{A M^{2}}{\sqrt{r}} \sum_{m=0}^{M-1} s_{T}\left(t-\frac{2 r}{c}\right)
$$

In general, the target location is unknown, i.e. $\theta_{0}$ is unknown. The coordinates used in the radar system is the polar coordinates. The coarse estimation of $\theta_{0}$ is implemented by using the $\theta_{0}$ sweeping method. That's to say, EUSR will scan through $0^{\circ}$ to $180^{\circ}$ by incrementing $\theta_{0}$ by $1^{\circ}$ for each step, until the maximum received energy is obtained. $\max \left\{E_{\mathrm{R}}\left(\theta_{0}\right)\right\}$ is the maximum received energy by the definition: 


$$
E_{R}\left(\theta_{0}\right)=\int_{t_{p}}^{t_{p}+t_{0}}\left|s_{R}\left(t, \theta_{0}\right)\right|^{2} d t
$$

Figure 11 is an example of the transmitted signal and received signal pair captured in our experiment. By mapping the time of flight into distance space, we can achieve the image of a scanned specimen.

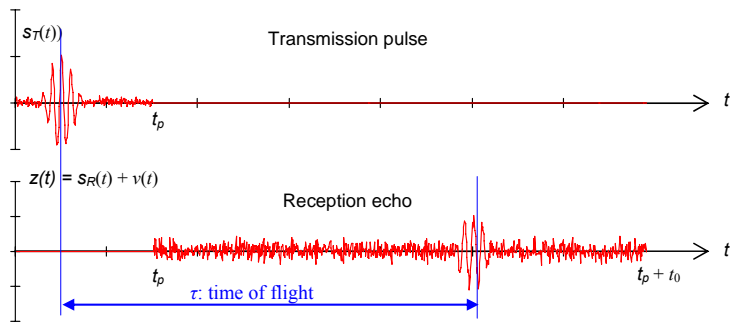

Figure 11: the transmitted signal and the received signal

The laboratory experiment setup is shown in Figure 12. The specimens are 1220-mm long, 1-mm thick square aluminum plates with an 8-element 7-mm x 7-mm PWAS array at the center. The damage is simulated by through-plate cracks 14 $\mathrm{mm}$ long and $0.5 \mathrm{~mm}$ wide. Figure 13 shows the mapping image of a specimen with two cracks at $67^{\circ}$ and $117^{\circ}$ in front of the PWAS array respectively.

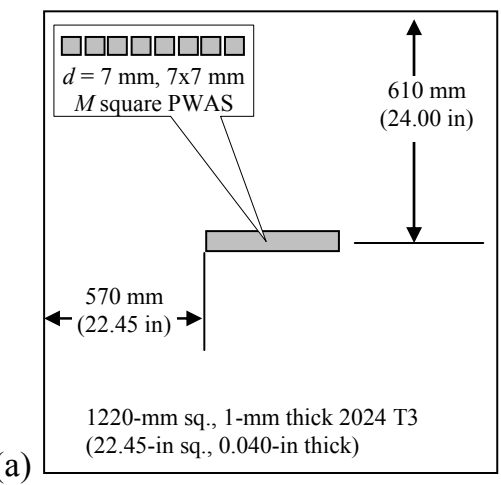

(b)

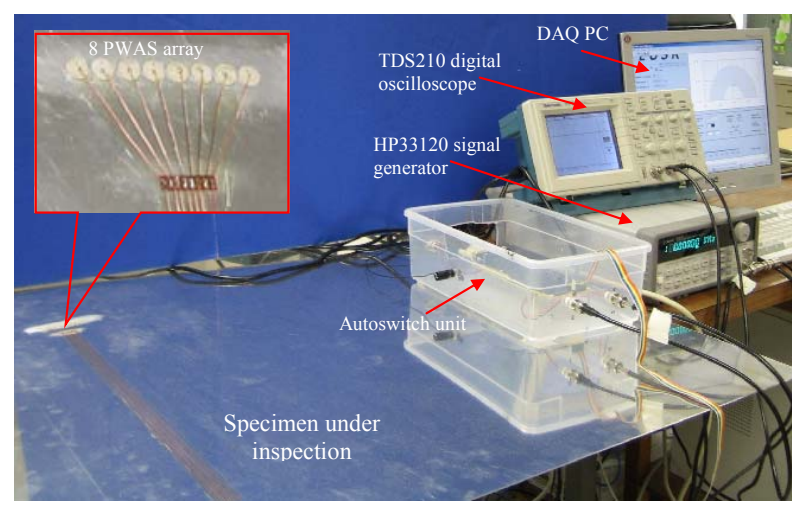

Figure 12: EUSR experiment using an 8 element PWAS array: (a) specimen layout; (b) experiment setup

\section{MINI-ARRAY DESIGNING AND IMPLEMENTATION}

In the optimization discussion, we concluded that to have better behaved beamforming of the PWAS phased array, more sensors

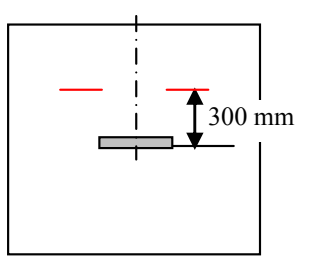

(a) (b)

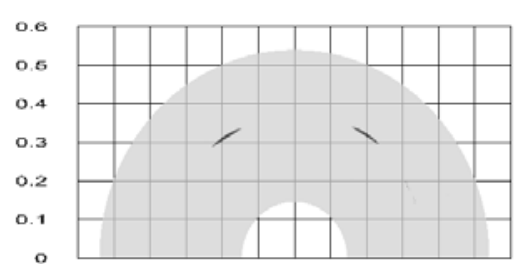

Figure 13: EUSR experiment on a specimen having two offside cracks at $67^{\circ}$ and $117^{\circ}$ : (a) specimen schematic; (b) EUSR mapped image

and/or small $d / r$ value are desired. Increasing number of sensors is no doubt the easier way to achieve obvious improvement. However, this will result in the complexity of the wiring and larger size of phased array, especially when the phased array is applied to specimens with compact and/or complex geometry where space is limited.

Hence, we proposed a design of using mini-array consisting of scale-down PWAS. The dimension of the previously used regular PWAS, either square or round, is $7-\mathrm{mm}$. We then scale them down to $5-\mathrm{mm}$ only. By this means, the complete size of the array shrinks by at least (7-mm $M-5-\mathrm{mm} \cdot M$ ). If $M=8$, this will be $16-\mathrm{mm}$. The mini- PWAS array makes our phased array further suitable for the inspection of small structures with complex geometry therefore.

\subsection{Frequency tuning of the mini-array: the sweet triple point}

One concern in the mini-PWAS array is, the ratio of $d / \lambda$ (or $d / \lambda$ ) has to remain constant, 0.5 . With smaller spacing $d$ (or smaller dimension $a$ ) the wavelength $\lambda$ of the excitation signal needs to be decreased as well.

Smaller wavelength also means an excitation with higher frequency is desired. In order to avoid Lamb wave dispersion in higher frequency range caused by complicated wave mode composition [8], a proper excitation frequency is necessary for the function of phased array [9]. Figure 14 shows the group velocity of Lamb waves in the Aluminum-2024-T3 specimen of 3-mm thickness.

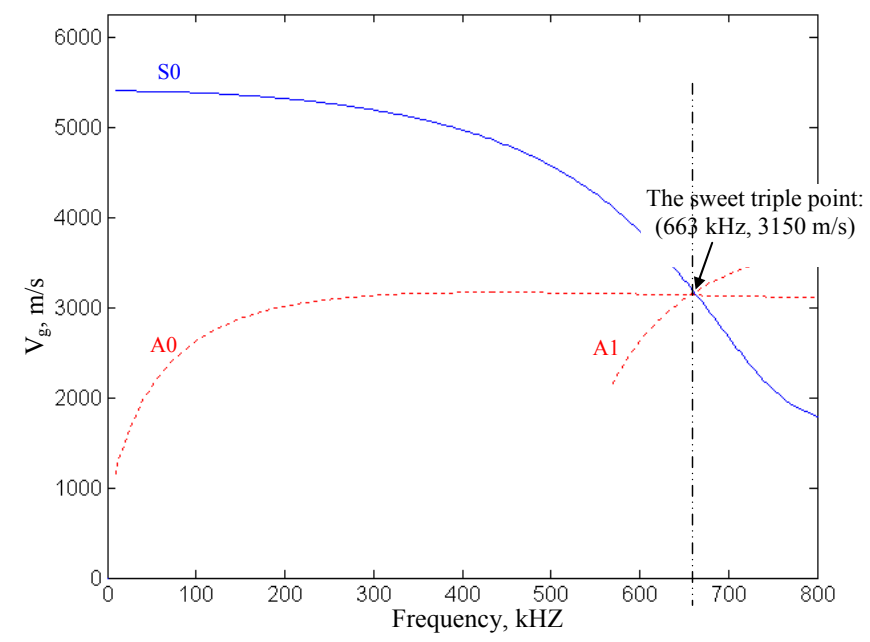

Figure 14: Lamb wave group velocity of Aluminum-2024-T3 of 3-mm thickness (Bottai and Giurgiutiu 2005) 
Within the illustrated frequency range of $0 \sim 800 \mathrm{kHz}$, there are 3 modes of Lamb wave existing, S0, A0 and A1. For most frequencies, the 3 modes travel at a different speed, which is not wanted for good imaging. However, from the plot we see, around the frequency $663 \mathrm{kHz}$, there is a "sweet point" where the 3 modes meet each other, i.e., have the same group velocity $(3150 \mathrm{~m} / \mathrm{s})$. By using this frequency as the excitation frequency for our mini-array, we can optimally keep the wave packet from separating.

\subsection{Experimente testing}

An Aluminum-2024-T3 specimen (150-mmx460-mm, 3-mm thick) with a through hole of $6-\mathrm{mm}$ is installed with regular 8 PWAS array and mini array with 10 small PWAS on each side (Figure 15). The hole is $80-\mathrm{mm}$ away from the arrays.

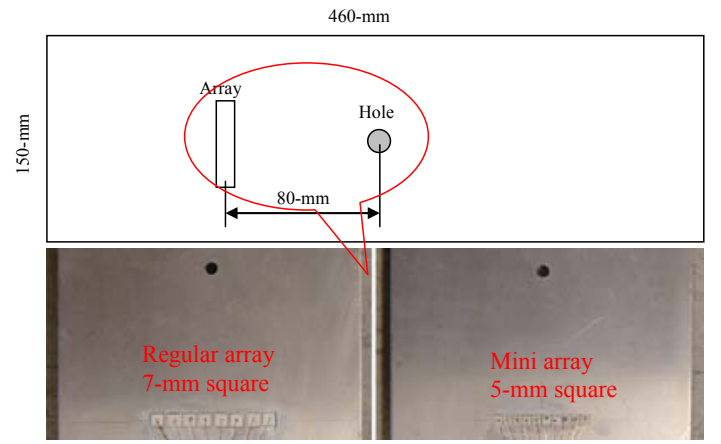

Figure 15: experimentation for comparing the detectionability of regular PWAS (7-mm sq.) and mini-PWAS (5-mm) array.

Considering the lateral and boundary reflection, damping material is applied along the boundary to absorb the reflections so that the echoes collected by the array are solely due to the hole defect. Figure 16 is the images of phased array inspection using the regular PWAS and mini PWAS respectively. The image of mini array (Figure 16b) indicates there is a defect at $80 \mathrm{~mm}$ perpendicular to the array which correctly shows the real situation while the image of the regular array has certain deviation (it indicates the defect is located $100 \mathrm{~mm}$ away). Also, We notice the mini array has a relatively smaller blind area compared to the regular array, i.e., the mini array can check the area much closer to the array. These results confirm the idea of using a scaled down phased array.

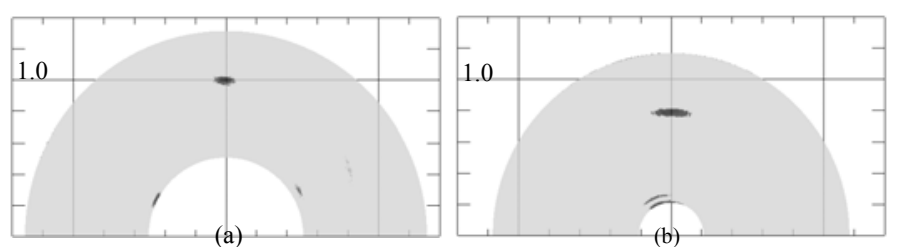

Figure 16: images of the inspection using regular PWAS (7-mm sq.) and mini-PWAS (5-mm) array: (a) EUSR image using 7-mm PWAS; (b) EUSR image using 5-mm PWAS

\section{CONCLUSIONS}

In this paper, generic formulation for phased array directional beamforming was obtained by using the exact traveling wave's formulation without the limiting parallel-rays assumption. Then the beamforming and lobe steering of 1-D linear PWAS Lamb wave phased array was researched with extensive simulation concerning the affects of several parameters including: (1) number of sensors; (2) spacing between sensors; (3) steering angles. Large number of sensors, smaller spacing (or smaller array size), or particular steering angle can lead to optimal beamforming. But the optimization on the other hand is limited by the installation such as wiring concerns or available space. Simulation results show that a well behaved directional PWAS phased array can be achieved with judicious array design. Furthermore, a mini-PWAS array was proposed for the damage detection of compact structures with complicated geometry. Frequency tuning becomes a serious issue for this array and we suggest using a sweet triple point in the Lamb-wave mode plot. Our preliminary experiment on a small plate with hole defect shows improved detection with the mini PWAS array.

With the generic phased array formulation, we envision to propose more array design for more powerful phased array damage detection. Further theoretical research on the optimization is also carrying on. Complicated specimens will be employed to verify the application of the mini PWAS array.

\section{ACKNOWLEDGMENTS}

Support from the Air Force Research Lab through UTC Contract \#03-S470-033-C1 of F33615-01-D-5801 is thankfully acknowledged.

\section{REFERENCES}

1 Wooh, S.C. and Shi (1999), Y., "Optimum Beam Steering of Linear Phased Arrays", Journal of Wave Motion,"

2 J.L Rose, "Recent Advances in Guided Wave NDE," 1995 IEEE Ultrasonics Symposium Proceedings, (Piscataway, NJ: IEEE, 1995), pp. 761-770.

3 J.L. Rose, "A Baseline and Vision of Ultrasonic Guided Wave Inspection Potential," ASME J. Pressure Vessel Technology: Special Issue on Nondestructive Characterization of Structural Materials, 124 (3) (August 2002), pp. 273-282

4 I.A. Viktorov, Rayleigh and Lamb Waves (New York: Plenum Press, 1967).

5 J.L. Rose, Ultrasonic Waves in Solid Media (Cambridge, U.K.: Cambridge University Press, 1999).

6 Giurgiutiu, V., Bao, J. (2004), "Embedded-Ultrasonics Structural Radar for In-Situ Structural Health Monitoring of Thin-Wall Structures", Structural Health Monitoring - an International Journal, Vol. 3, NO. 2, pp. 121-140

7 Johnson, D.H. and Dudgeon, D.E. (1993), Array signal processing: Concepts and Techniques, PTR Prentice-Hall Inc., Upper Saddle River, NJ 07458

8 Graff, K.F. (1975), Wave Motion in Elastic Solids, Oxford University Press

9 Bottai, G., Giurgiutiu, V. (2005), "Simulation of the Lamb Wave Interaction between Piezoelectric Wafer Active Sensors and Host Structure", SPIE's $12^{\text {th }}$ International Symposium on Smart Structures and Materials and $10^{\text {th }}$ International Symposium on NDE for Health, Monitoring and Diagnostics, Sensors and Smart Structures Technologies for Civil, Mechanical, and Aerospace Systems Conference, San Diego, CA, 7-10 March, 2005, paper \# 5765-29 